\title{
Polarised Drell-Yan at COMPASS
}

\author{
Riccardo Longo ${ }^{1,2}$ \\ on behalf of the COMPASS Collaboration \\ email: riccardo.longo@cern.ch \\ ${ }^{1}$ INFN sezione di Torino \\ ${ }^{2}$ Università di Torino
}

\begin{abstract}
The COMPASS experiment at CERN took the polarised Drell-Yan data in 2015. The muon pairs originating from $190 \mathrm{GeV} / c$ pion collisions with polarised protons provide a way of accessing the transverse momentum dependent parton distribution functions of the nucleon. The study of the azimuthal spin asymmetries in Drell-Yan complements a wealth of results already obtained from transversely polarised semi-inclusive deep inelastic scattering at COMPASS.

The first results from the polarised Drell-Yan measurements will be shown in the context of the previously obtained SIDIS results. The expected impact of these data will be discussed, as well as prospects for future Drell-Yan related studies.
\end{abstract}

\section{Introduction}

One of the main purposes of the COMPASS experiment at CERN is the exploration of the transverse spin structure of the nucleon through the study of spin (in)dependent azimuthal asymmetries of Semi Inclusive Deep Inelastic Scattering (SIDIS) and Drell-Yan (DY) processes. The lepton polarisationindependent part of the transversely polarised SIDIS cross-section contains several Transverse Spin Asymmetries (TSAs). Within the QCD improved parton model approach, these observables are interpreted in terms of convolutions of Transverse Momentum Dependent (TMD) Parton Distribution Functions (PDFs) and Fragmentation Functions (FFs). The cross-section of pion induced DY leptonpair production off a transversely polarised nucleon $\left(\pi^{-}+p^{\uparrow} \rightarrow \mu^{+}+\mu^{-}+X\right)$ also includes analogous TSAs, interpreted in terms of convolutions of TMDs, one for the target nucleon and the other for the beam hadron. FFs are absent here.

The COMPASS experiment is currently the only facility studying the nucleon spin structure by measuring both SIDIS and DY processes, using essentially the same experimental setup and exploring the same kinematic domain. Thus COMPASS has a unique possibility to test universality and other predicted key-features of TMDs, like the sign change of the Sivers asymmetry when extracted in SIDIS or in DY processes [1].

\section{SIDIS and DY processes}

At the leading twist QCD and taking into account the polarisation and intrinsic transverse momentum $\left(k_{T}\right)$ of the quark, as well as the polarisation of its parent nucleon, the hadron structure is parametrized 
in terms of eight TMDs for each quark flavour. These non-perturbative functions describe the correlations between the quark spin, its $k_{T}$ and the spin of the nucleon. Four of these TMDs, namely the Sivers, the Boer-Mulders, the transversity and the pretzelosity functions, can be accessed at COMPASS by measuring the TSAs of both SIDIS and DY processes.

The lepton polarisation-independent part of the cross-section for the lepton off transversely polarised nucleon SIDIS process [2-4] can be written, at leading-twist, as:

$$
\begin{aligned}
\sigma^{S I D I S} \propto\{1 & +\varepsilon A_{U U}^{\cos \left(2 \phi_{h}\right)} \cos \left(2 \phi_{h}\right)+S_{T}\left[A_{U T}^{\sin \left(\phi_{h}-\phi_{S}\right)} \sin \left(\phi_{h}-\phi_{S}\right)\right. \\
& \left.\left.+\varepsilon A_{U T}^{\sin \left(\phi_{h}+\phi_{S}\right)} \sin \left(\phi_{h}+\phi_{S}\right)+\varepsilon A_{U T}^{\sin \left(3 \phi_{h}-\phi_{S}\right)} \sin \left(3 \phi_{h}-\phi_{S}\right)\right]\right\}
\end{aligned}
$$

where $S_{T}$ is the target transverse polarisation, $\varepsilon=\left(1-y-\frac{1}{4} \gamma^{2} y^{2}\right) /\left(1-y+\frac{1}{2} y^{2}+\frac{1}{4} \gamma^{2} y^{2}\right)$ is the ratio of longitudinal and transverse photon fluxes, $\gamma=2 M x / Q$ and $\phi_{h}$ and $\phi_{S}$ are the azimuthal angles of the produced hadron and of the nucleon spin, respectively (see Fig. 1). Each azimuthal modulation is associated to a TSA $A_{B T}^{\omega_{i}\left(\phi_{h}, \phi_{S}\right)}$ term ${ }^{1}$, proportional to the convolution of the corresponding TMD PDF and TMD FF. For example, the Sivers asymmetry, $A_{U T}^{\sin \left(\phi_{h}-\phi_{S}\right)}$, gives access to the convolution of the Sivers function with the unpolarised TMD FF describing the final hadron, $f_{1 T}^{\perp q} \otimes D_{1 q}^{h}$.

Using similar notations and another set of angular variables, defined in the so-called Collins-Soper (CS) frame - see Fig. 2, left panel - and in the target rest frame - see Fig. 2, right panel - the LO crosssection for the single-polarised DY process [5] can be written as:

$$
\begin{aligned}
\sigma^{D Y} \propto\{1 & +\cos ^{2}\left(\theta_{C S}\right)+\sin ^{2}\left(\theta_{C S}\right) A_{U}^{\cos \left(2 \varphi_{C S}\right)} \cos \left(2 \varphi_{C S}\right) \\
+ & S_{T}\left[\left(1+\cos ^{2}\left(\theta_{C S}\right)\right) A_{T}^{\sin \phi_{S}} \sin \phi_{S}+\sin ^{2}\left(\theta_{C S}\right) A_{T}^{\sin \left(2 \varphi_{C S}+\varphi_{S}\right)} \sin \left(2 \varphi_{C S}+\varphi_{S}\right)\right. \\
& \left.\left.+\sin ^{2}\left(\theta_{C S}\right) A_{T}^{\sin \left(2 \varphi_{C S}-\varphi_{S}\right)} \sin \left(2 \varphi_{C S}-\phi_{S}\right)\right]\right\}
\end{aligned}
$$

where $S_{T}$ is the target transverse polarisation. Similarly to the SIDIS case, each azimuthal modulation is associated to a TSA, the $A_{T}^{\omega_{i}\left(\varphi_{C S}, \varphi_{S}\right)} \operatorname{term}^{2}$, proportional to the convolution of the corresponding

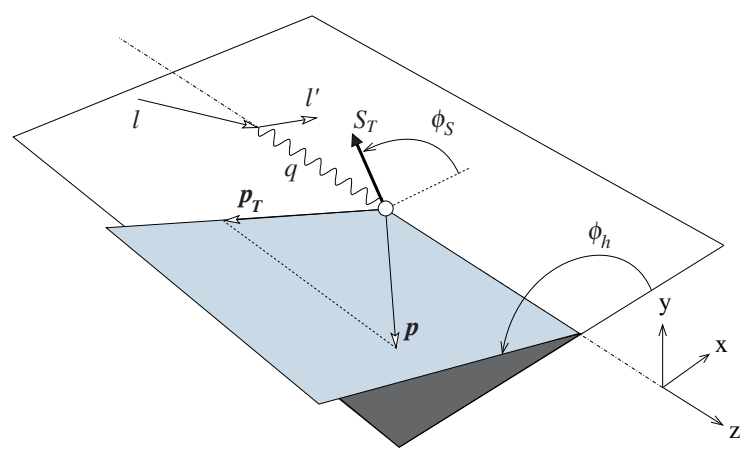

Figure 1: Definition of $\phi_{h}$ and $\phi_{S}$ angles in the SIDIS case.

\footnotetext{
${ }^{1}$ In $A_{B T}^{\omega_{i}\left(\phi_{h}, \phi_{S}\right)}$ the superscript of the asymmetry indicates the corresponding modulation, the first subscript the polarisation of the beam while the second one the polarisation of the target (U - "Unpolarised", T - "Transverse")

${ }^{2}$ For the single-polarised case just the target polarisation is being quoted as a subscript (U - "Unpolarised", T - "Transverse").
} 

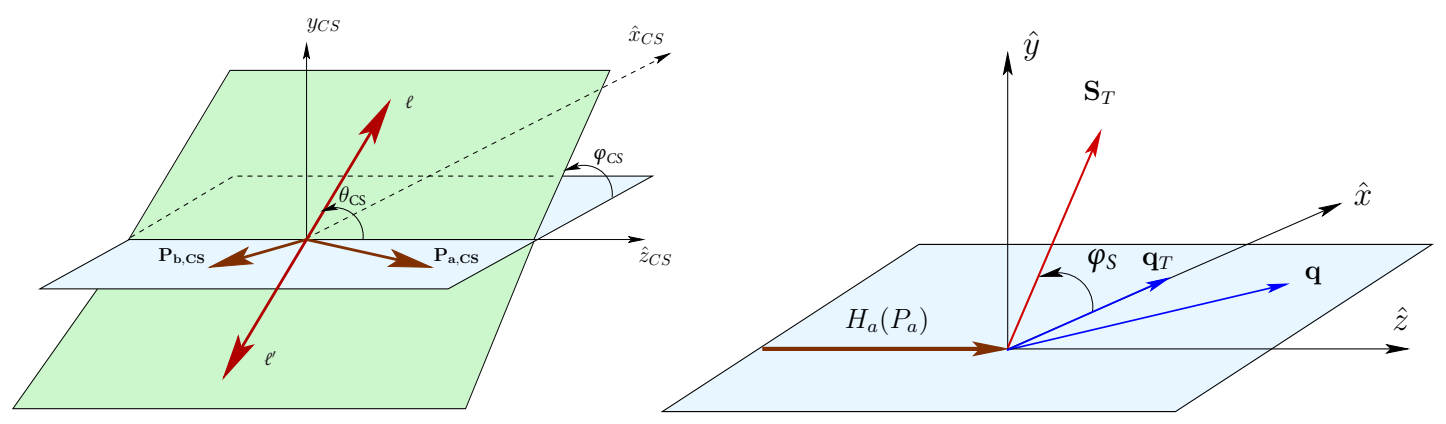

Figure 2: $\varphi_{C S}$ and $\theta_{C S}$ definition in Collins-Soper frame (left panel) and $\varphi_{S}$ definition in the target rest frame (right panel) for DY.

TMD PDFs of the beam and of the target. For example, in $\pi^{-}+p^{\uparrow}$ collisions as in the COMPASS DY case, the Sivers asymmetry $A_{T}^{\sin \left(\varphi_{S}\right)}$ is giving access to a convolution of the unpolarised TMD PDF of the $\pi^{-}$with the Sivers function of the target proton, $f_{1, \pi}^{q} \otimes f_{1 T, p}^{\perp q}$.

A list of nucleon TMD PDFs and the corresponding LO TSAs in SIDIS and DY measured at COMPASS can be found in Tab. 1 .

Table 1: Leading Order asymmetries in DY and SIDIS and related nucleon TMDs.

\begin{tabular}{|c|c|c|}
\hline Drell-Yan & Proton TMD PDF & SIDIS \\
\hline \hline$A_{U}^{\cos \left(2 \varphi_{C S}\right)} \propto h_{1, \pi}^{\perp q} \otimes h_{1, p}^{\perp q}$ & Boer-Mulders & $A_{U U}^{\cos \left(2 \phi_{h}\right)} \propto h_{1, p}^{\perp q} \otimes H_{1 q}^{\perp h}$ \\
\hline$A_{T}^{\sin \left(\varphi_{S}\right)} \propto f_{1, \pi}^{q} \otimes f_{1 T, p}^{\perp q}$ & Sivers & $A_{U T}^{\sin \left(\phi_{h}-\phi_{S}\right)} \propto f_{1 T}^{\perp q} \otimes D_{1 q}^{h}$ \\
\hline$A_{T}^{\sin \left(2 \varphi_{C S}-\varphi_{S}\right)} \propto h_{1, \pi}^{\perp q} \otimes h_{1, p}^{q}$ & Transversity & $A_{U T}^{\sin \left(\phi_{h}+\phi_{S}\right)} \propto h_{1}^{q} \otimes H_{1 q}^{\perp h}$ \\
\hline$A_{T}^{\sin \left(2 \varphi_{C S}+\varphi_{S}\right)} \propto h_{1, \pi}^{\perp q} \otimes h_{1 T, p}^{\perp q}$ & Pretzelosity & $A_{U T}^{\sin \left(3 \phi_{h}-\phi_{S}\right)} \propto h_{1 T}^{\perp q} \otimes H_{1 q}^{\perp h}$ \\
\hline
\end{tabular}

\section{SIDIS and DY studies at COMPASS}

COMPASS collected a large amount of SIDIS data during its Phase I (2002-2011), making use of a longitudinally polarised muon beam and transversely polarised $\mathrm{NH}_{3}$ and ${ }^{6} \mathrm{LiD}$ targets. SIDIS TSAs have been extensively studied and, for example, Sivers asymmetry extracted from 2007 and 2010 SIDIS data [6]. Compared with the HERMES results, the Sivers asymmetry measured by COMPASS is slightly smaller. This systematic difference was interpreted as an effect of a different $Q^{2}$ coverage of the two experiments. Therefore a dedicated analysis of the COMPASS SIDIS data was performed in four $Q^{2}$ kinematic regions, the same as for the COMPASS Drell-Yan analysis:
i. $1<Q^{2} /(\mathrm{GeV} / c)^{2}<4 \rightarrow$ low dimuon mass, $M_{\mu \mu}$, range
ii. $4<Q^{2} /(\mathrm{GeV} / c)^{2}<6.25 \rightarrow$ intermediate mass range
iii. $6.26<Q^{2} /(\mathrm{GeV} / c)^{2}<16 \rightarrow J / 4$ mass range
iv. $16<Q^{2} /(\mathrm{GeV} / c)^{2}<81 \rightarrow$ high mass range 

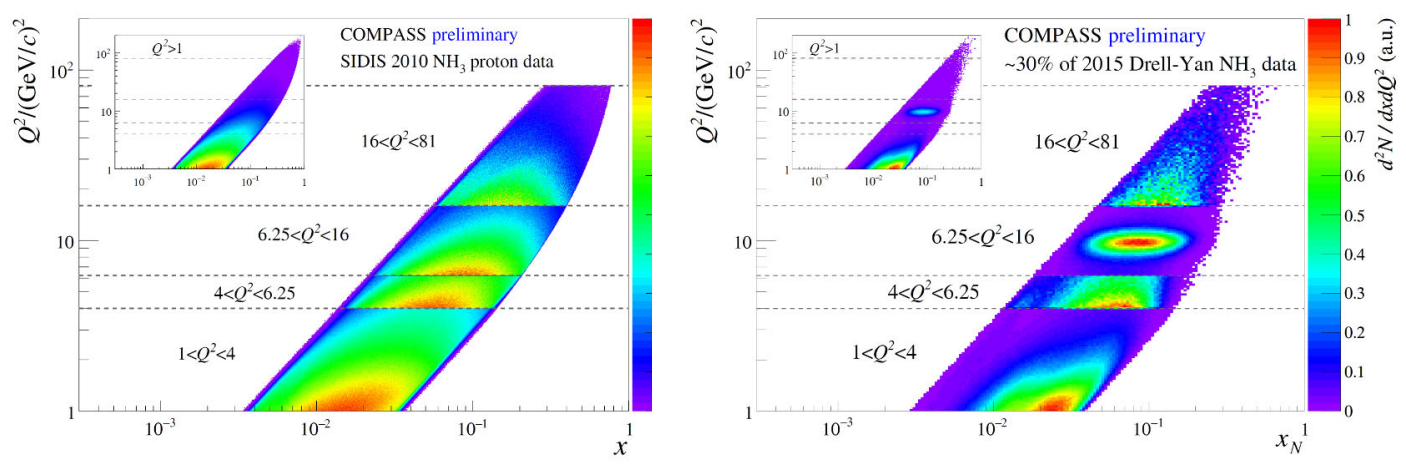

Figure 3: $Q^{2}$ vs $x_{N}$ coverage of SIDIS 2010 (left) and DY 2015 (right) data taking. Both distributions are divided into the same four $Q^{2}$ ranges. Inserts show the overall distributions without dedicated normalization for each $Q^{2}$ range.

A comparison of the SIDIS $\left(Q^{2}, x_{N}\right)$ kinematical phase-space with the Drell-Yan measurements one is presented in Fig. 3, where $x_{N}$ represents the nucleon Bjorken variable.

Among the four DY $Q^{2}$-ranges the most convenient for the TSAs studies is the high mass range. First, in this range the requirement of TMD factorization that the transverse momentum of the hadron in SIDIS or the dimuon in DY has to be much smaller than $Q$, is satisfied best. Second, for the COMPASS measurements in the high mass range, both SIDIS and DY cross-sections are dominated by the contribution of valence u-quarks inside the nucleon, where the Sivers TMD PDF is predicted to reach its maximum [7], [8]. Third, in this region, a background contribution is negligible.

SIDIS TSAs extracted from the four $Q^{2}$ DY-ranges have recently been submitted for publication by COMPASS [9]. The results for the SIDIS TSAs extracted at the high mass hard scale are summarized in Fig.4, where positive hadrons show a clear positive signal for the Sivers asymmetry. The Collins

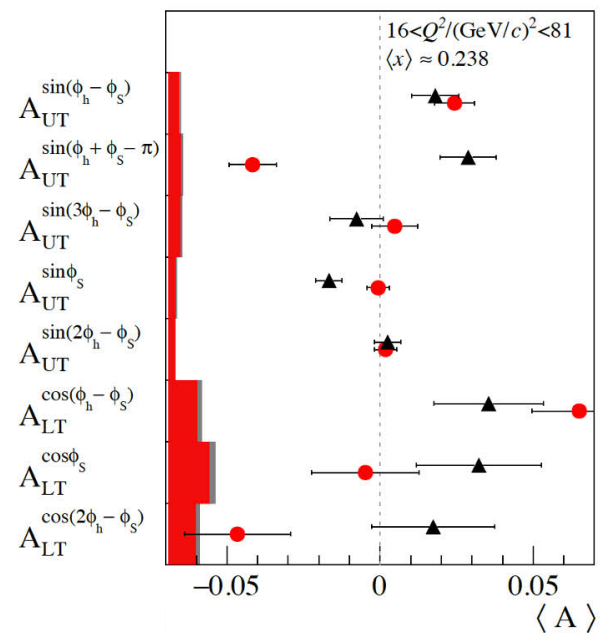

Figure 4: Mean SIDIS TSAs in the Drell-Yan high mass range. 
TSA, which gives access to the transversity TMD, is significantly negative, while the pretzelosityrelated asymmetry is compatible with zero.

\section{DY data taking}

In 2015 COMPASS collected the first polarised DY data in a fixed target experiment, using a high energy negative pion beam $(190 \mathrm{GeV} / c)$ impinging on a transversely polarised $\mathrm{NH}_{3}$ target, composed of two cells, oppositely polarised. A hadron absorber was placed downstream of the $\mathrm{NH}_{3}$ target, to stop the huge flux of secondary hadrons produced in the $\pi^{-}+p$ collisions as well as the noninteracting pion beam. For this purpose, a tungsten (W) plug $120 \mathrm{~cm}$ long was installed inside the absorber, centred on the beam line axis. To profit from the non-interacting pion beam the $\mathrm{W}$ plug is being used, together with a thin aluminum target installed upstream the $\mathrm{W}$, as a source of unpolarised DY events.

Around four months of data taking were performed in 2015, and the data are now being analyzed. Preliminary kinematical distributions, obtained from $\sim 30 \%$ of these data, are shown in Figs. 5 to 9 . In Fig. 5 the position of the DY interaction point along the beam axis is presented. The shaded regions (blue online) identify, from left to right, the two $\mathrm{NH}_{3}$ target cells, the aluminum and the tungsten targets. As it can be seen, in the high mass range the $\mathrm{NH}_{3}$ target cells are rather well separeted. In Fig. 6 we present the dimuon mass spectra ${ }^{3}$, while in Figs. 7 and 8 the Feynman variable, $x_{F}$, and $q_{T}$ distribution for high mass DY events are shown. The kinematical regions currently selected for the analysis are shaded (blue online). Fig. 9 shows the $\left(x_{N}, x_{\pi}\right)$ distribution for selected DY events in the high mass range.

Given the error obtained from the analysis of this $30 \%$ fraction of 2015 data, an uncertainty of about $4 \%$ for the Sivers asymmetry is expected from the analysis of the whole 2015 data sample. In the case of another DY run of 140 days in $2018^{4}$, a precision of $\sim 2-3 \%$ will be achievable. These values are plotted in Figs. 10 and 11 together with the phenomenological predictions available in Refs. [10-13].

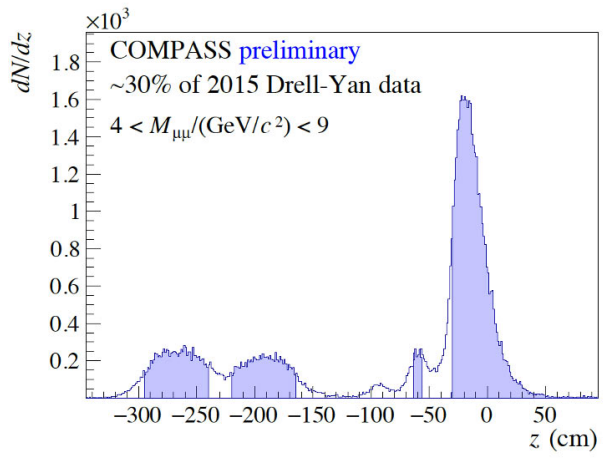

Figure 5: $z$ vertex distribution of DY events from the high mass range.

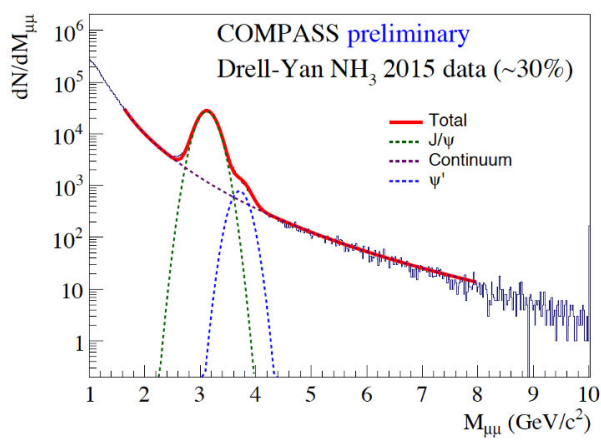

Figure 6: Dimuon mass distribution for DY events.

\footnotetext{
${ }^{3}$ Neglecting muon mass, $M_{\mu \mu} \sim Q$.

${ }^{4}$ Presently under an approval procedure.
} 


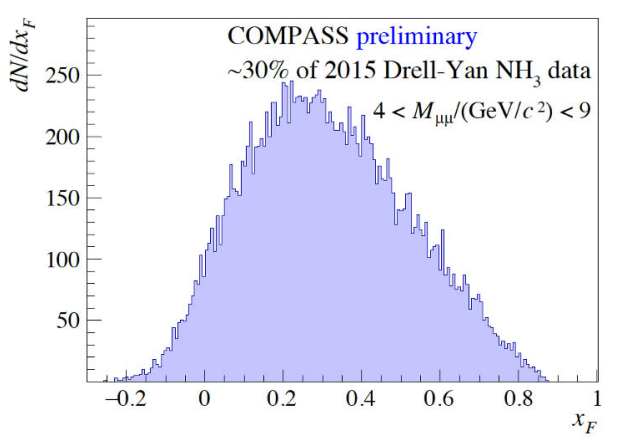

Figure 7: $x_{F}=x_{\pi}-x_{N}$ of DY events in the high mass range.

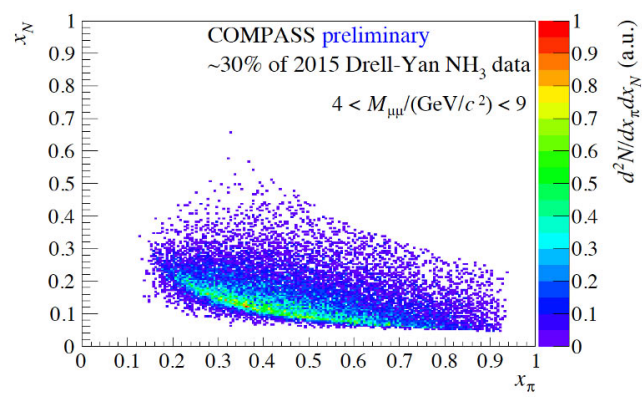

Figure 9: $x_{N}$ vs $x_{\pi}$ distribution for DY events in the high mass range.

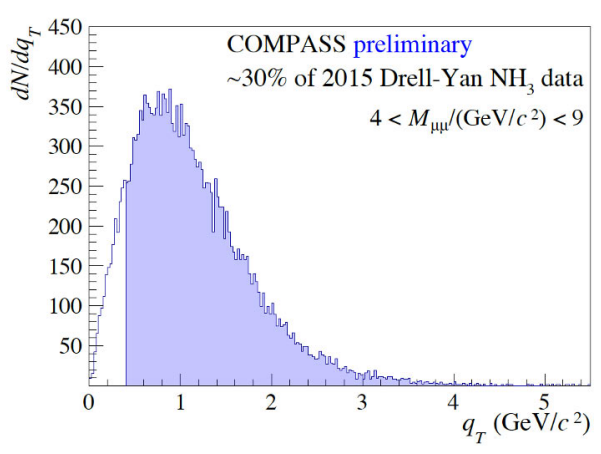

Figure 8: $q_{T}$ of DY events in the high mass range.

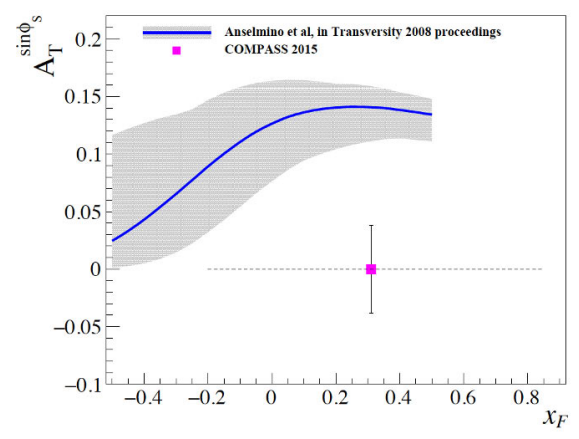

Figure 10: Projected Sivers asymmetry uncertainty in the high mass range after the analysis of the whole 2015 data sample.

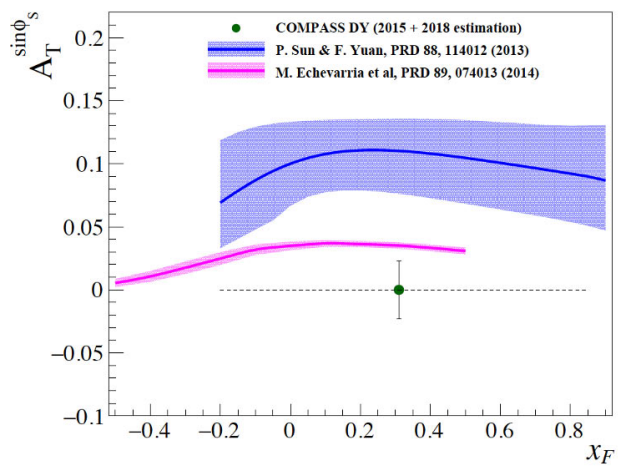

Figure 11: Projected Sivers asymmetry uncertainty in the high mass range for two years of data taking: 2015 (achieved) and 2018 (data taking to be approved). 


\section{Conclusions}

Last year COMPASS collected its first transversely polarised DY data. COMPASS has recently provided the first input for future direct SIDIS-DY studies [9]. Combining these results with the upcoming results of 2015 data analysis, COMPASS will have the opportunity to access TMDs via the two processes measured using essentially the same experimental setup and exploring the same $\left(Q^{2}, x_{N}\right)$ kinematical range, having the possibility to test TMDs key features like universality and the Sivers PDF sign change.

\section{References}

[1] J.C. Collins, Phys. Lett. B536, 43 (2002), hep-ph/0204004

[2] A. Kotzinian, Nucl. Phys. B441, 234 (1995), hep-ph/9412283

[3] M. Anselmino et al., Phys. Rev. D83, 114019 (2011), hep-ph/1101. 1011

[4] A. Bacchetta et al., JHEP 02, 093 (2007), hep-ph/0611265

[5] S.Arnold, A.Metz and M.Schlegel, Phys. Rev. D79, 034005 (2009), hep-ph/0809 . 2262

[6] C. Adolph et al. (COMPASS), Phys. Lett. B717, 383 (2012), hep-ex/1205 . 5122

[7] M. Anselmino et al., Phys. Rev. D72, 094007 (2005), [Erratum: Phys. Rev.D72,099903(2005)], hep-ph/0507181

[8] M. Anselmino et al., Eur. Phys. J. A39, 89 (2009), hep-ph/0805 . 2677

[9] C. Adolph et al. (COMPASS), to appear in Phys. Lett. B (2016), hep-ex/1609.07374

[10] M. Anselmino et al., AIP Conf. Proc. 1105, 262 (2009)

[11] M. Anselmino et al., Phys. Rev. D79, 054010 (2009), hep-ph/0901. 3078

[12] P.Sun and F.Yuan, Phys. Rev. D88, 034016 (2013), hep-ph/1304 . 5037

[13] M.G.Echevarria et al., Phys. Rev. D89, 074013 (2014), hep-ph/1401. 5078 